\title{
PATH TRACKING FOR AN AUTONOMOUS VEHICLE DURING EMERGENCY CONDITION
}

\author{
ZULKARNAIN $^{1}{ }^{*}$, ISMAIL THAMRIN ${ }^{1}$, FIRMANSYAH BURLIAN, INDAH NOVIANTY ${ }^{2}$ \\ ${ }^{1}$ Dept. of Mechanical Engineering, Universitas Sriwijaya, Palembang, Indonesia \\ ${ }^{2}$ Balai Pengelola Transportasi Darat Wilayah VII Sumsel-Babel, Kementrian Perhubungan, \\ Palembang, Indonesia
}

*Corresponding author: zulkarnain@ft.unsri.ac.id

(Received: 21 March 2021; Accepted: 30 May 2021; Published on-line: 1 July 2021)

\begin{abstract}
An autonomous vehicle's primary function is detecting and tracking the road course precisely and correctly without a driver's assistance. As a result, implementing appropriate controllers is critical for improving the vehicle's stability and movement responsiveness. The performance of adaptive Stanley controlled is evaluated in this paper using numerical simulations. The Stanley controller's most common geometric controller for vehicle path tracking algorithms is compared based on their trajectory tracking analyses on various vehicle speed maneuvers. Stanley calculates steering based on the difference between the vehicle's lateral position and heading angle. The difference between desired coordinates and present coordinates of the vehicle along the path is used to calculate lateral, longitudinal, and vehicle heading orientation angle using the future prediction control technique. The results demonstrate that the Stanley controller outperforms the emergency trajectory with more consistent trajectory tracking and steady-state error.
\end{abstract}

KEY WORDS: Autonomous Vehicle, Stanley Controller, PSO, Lateral Error, Yaw Error.

\section{INTRODUCTION}

The vehicle dynamic control on the road path by regulating the vehicle lateral dynamic motion is essential in defining the vehicle's stability and accuracy in response to the path tracking mechanism in developing an autonomous vehicle. Vehicle accidents are frequently caused by a human mistake in the driving task, which can be reduced if the driving duty is done autonomously by a machine [1-3]. Therefore, an advanced control technique must be used to drive in various road conditions, including straight, curved, and other terrain types. As an autopilot car capable of detecting the surrounding environment, making decisions, and navigating itself with an anti-collision system, the autonomous vehicle will replace the driver.

Autonomous vehicles require sophisticated environmental recognition, path planning, motion tracking, and real-time allocation and need linear and nonlinear vehicle models based on dynamic and kinematic characteristics to solve trajectory tracking problems [5-6]. Each controller will be installed on the vehicle to follow the reference road characteristics of straight lines, sharp corners, and obstacle avoidance.

There are many types of controls for testing the performance of autonomous vehicles, such as using future prediction control, PID, fuzzy logic, or even modeling using state-space models. In this scenario, the future prediction control approach provides feedback on the vehicle's future position as the intended path compared to its present coordinate. The heading angle error, longitudinal error, and lateral error are calculated from the global coordinates of reference 
points to the current vehicle's local position [7]. Certain variables or parameters must be compared to guarantee vehicle stability to fulfill the control objectives. Those variables include yaw rate and sideslip angle for a quick and exact reaction of required route tracking potential. In addition, a robust and precise route tracking algorithm with an appropriate controller is needed to achieve trajectory tracking amid unfavorable circumstances [8-10].

The effectiveness of steering control law on vehicle models is being investigated using primary controllers such as the Stanley Controller. The efficacy of the Stanley controller will be analyzed at a speed of $10 \mathrm{~m} / \mathrm{s}-25 \mathrm{~m} / \mathrm{s}$. The accuracy of the proposed controller is measured by the resulting lateral and yaw errors. In addition to this, to get optimal accuracy, the controller gain will be calculated using the particle swarm optimization (PSO) algorithm.

\section{MODEL DYNAMIC VEHICLE}

The dynamic model is based on the two degrees of freedom (DOF) of a bicycle model, which primarily consists of yaw motion and lateral acceleration utilizing Newton's second equation of motion to describe the forces and moments acting on the vehicle body and tire.

This model is suitable for capturing the vehicle's lateral dynamics in low and mediumspeed environments. Because it does not have enough tire force style, this model is sufficient to be applied to the roadway in most autonomous vehicle maneuvering analyses. However, the linear model is not ideal in many designs and cases, following a reference path with a small turning radius or high-speed maneuvers. Fig. 1 and 2 demonstrate the typical automobile terminology used when modeling a vehicle and a linear bicycle model.

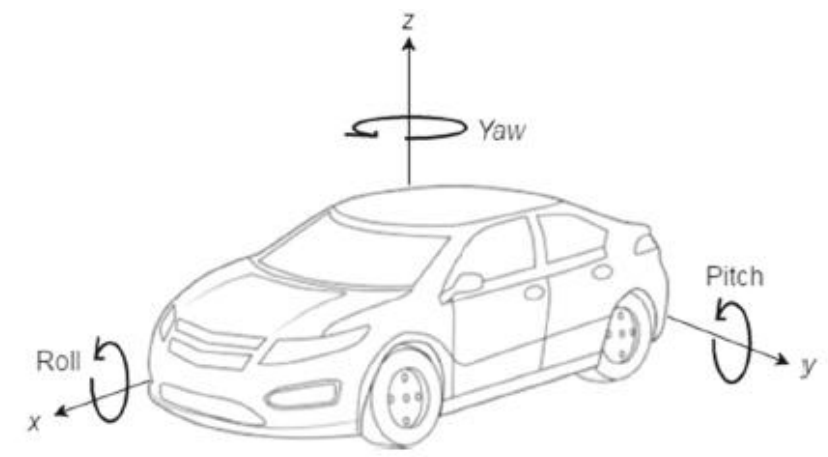

Fig. 1. Vehicle view and its standard nomenclature used [6]

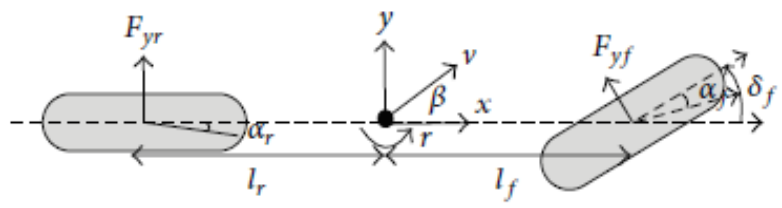

Fig. 2. Linear Bicycle Model [6]

Lateral Motion equation:

$$
m v\left(\beta^{\prime}+r\right)=\left(F_{y f}+F_{y r}\right)-r
$$

Yaw Motion equation:

$$
I_{z} r^{\prime}=l_{f} \cdot F_{y f}-l_{r} \cdot F_{y r}
$$


Since this simplest planar is a linearized model which all the forces are simplified, the vehicle model may be reduced into a state-space model representation by rearranging the yaw and lateral motion equations, as shown in Equation 3:

$$
\begin{aligned}
& \dot{x}=A x+B u \\
& y=C x+D u
\end{aligned}
$$

where A, B, C, D, and the vehicle parameters are classified as followed:

$$
\begin{aligned}
& A=\left[\begin{array}{cc}
\frac{\left(-C_{f}-C_{r}\right)}{m v} & -1+\frac{\left(C_{r} l_{r}-C_{f} l_{f}\right)}{m v^{2}} \\
\frac{\left(C_{\left.r l_{r}-C_{f} l_{f}\right)}\right.}{I_{z}} & \frac{-C_{f} l_{f}^{2}-C_{r} l_{r}^{2}}{I_{z} v}
\end{array}\right] \\
& B=\left[\begin{array}{c}
\frac{C_{f}}{m v} \\
\frac{C_{f} l_{f}}{I_{z}}
\end{array}\right] \\
& C=\left[\begin{array}{cc}
\frac{\left(-C_{f}-C_{r}\right)}{m} & \frac{\left(C_{r} l_{r}-C_{f} l_{f}\right)}{m v^{2}} \\
0 & 1
\end{array}\right] \\
& D=\left[\begin{array}{c}
\frac{C_{f}}{m} \\
1
\end{array}\right]
\end{aligned}
$$

The characteristics of the vehicle are based on the minivan that will be utilized in the analysis, vehicle parameter presented in Table 1. In the vehicle yaw stability control, the first equation of the linear state-space model, $x^{\prime}=[\beta, r]$, is used to create the sideslip angle and yaw rate response. Next, the actual vehicle's sideslip and yaw rate using the suggested controller will be compared to the desired response to determine the tracking control.

Table 1: Vehicle variables and parameters

\begin{tabular}{clll}
\hline Symbol & Unit & Values & Remarks \\
\hline$m$ & $\mathrm{~kg}$ & 2023 & vehicle mass \\
$I_{z}$ & $\mathrm{~kg} \cdot \mathrm{m}^{2}$ & 6286 & vehicle yaw inertia \\
$I_{r}$ & $\mathrm{~kg} \cdot \mathrm{m}^{2}$ & 900 & vehicle roll inertia \\
$l_{r}$ & $\mathrm{~m}$ & 1.90 & distance Centre of Gravity - rear axle \\
$l_{f}$ & $\mathrm{~m}$ & 1.26 & distance Centre of Gravity - front axle \\
$C_{r}$ & $\mathrm{~N} / \mathrm{rad}$ & 40200 & cornering stiffness of the rear tire \\
$C_{f}$ & $\mathrm{~N} / \mathrm{rad}$ & 62800 & cornering stiffness of the front tire \\
$v$ & $\mathrm{~km} / \mathrm{h}$ & 30 to 50 & vehicle velocity interval \\
\hline
\end{tabular}

In contrast to the linear vehicle model, the nonlinear vehicle model may be represented and stimulated like an actual vehicle for controller validation and assessment since all internal and external pressures are considered (Fig. 3). However, the tire forces are the most critical component that impacts the model since they disturb the vehicle's motion. 


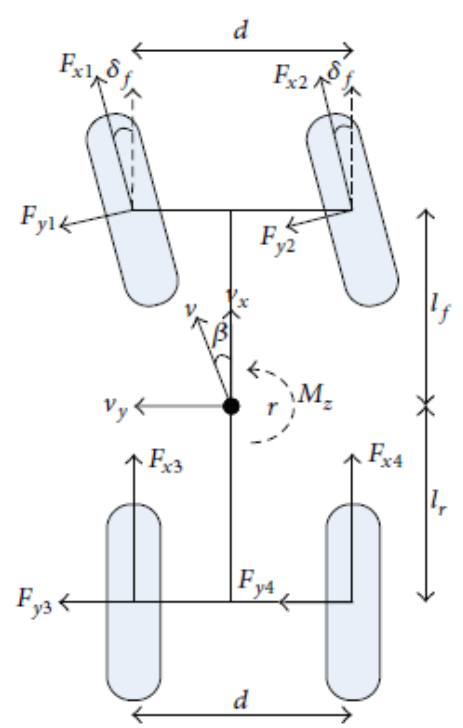

Fig. 3. Nonlinear vehicle model with 7 DOF [7]

The tire model provides the proper tire dynamics in the real world [9]. The vehicle's forces and moments are represented by the equations [10]:

$$
\begin{aligned}
& F_{x}=F_{x 1} \cos \delta_{f l}-F_{y 1} \sin \delta_{f l}+F_{x 2} \cos \delta_{f r}-F_{y 2} \sin \delta_{f r}+F_{x 3} \cos \delta_{r l}-F_{y 3} \sin \delta_{r l}+ \\
& F_{x 4} \cos \delta_{r r}-F_{y 4} \sin \delta_{r r} \\
& F_{y}=F_{x 1} \sin \delta_{f l}+F_{y 1} \cos \delta_{f l}+F_{x 2} \sin \delta_{f r}+F_{y 2} \cos \delta_{f r}+F_{x 3} \sin \delta_{r l}+F_{y 3} \cos \delta_{r l}+ \\
& F_{x 4} \sin \delta_{r r}+F_{y 4} \cos \delta_{r r} \\
& M_{z}=I_{s}\left(-F_{x 1} \cos \delta_{f l}+F_{y 1} \sin \delta_{f l}-F_{x 2} \cos \delta_{f r}+F_{y 2} \sin \delta_{f r}\right)+I_{s}\left(F_{x 3} \cos \delta_{r l}-\right. \\
& \left.F_{y 3} \sin \delta_{r l}+F_{x 4} \cos \delta_{r r}-F_{y 4} \sin \delta_{r r}\right)+I_{s}\left(F_{x 1} \sin \delta_{f l}+F_{y 1} \cos \delta_{f l}+F_{x 2} \sin \delta_{f r}+\right. \\
& \left.F_{y 2} \cos \delta_{f r}\right)+I_{s}\left(-F_{x 3} \sin \delta_{r l}-F_{y 3} \cos \delta_{r l}-F_{x 4} \sin \delta_{r r}-F_{y 4} \cos \delta_{r r}\right.
\end{aligned}
$$

$F x^{\prime} s$ lateral tire force is characterized as a linear function of tire cornering stiffness and tire sideslip angle for lower slip angle and slips ratio conditions. In contrast, $F y$ 's longitudinal tire force is given as a linear function of braking stiffness and tire slip ratio. Otherwise, the lateral and longitudinal tire forces show nonlinear behavior at increasing slip angles and slip ratios. While nonlinear vehicle models have varying degrees of freedom (DOF), throughout this case, it has seven DOF, which indicate the vehicle's dynamic motion and complexity. The seven degrees of freedom (DOF) are lateral, longitudinal, yaw, and four tire-wheel forces.

\section{KINEMATIC}

Kinematic motion is studying a vehicle's motion without respect for the vehicle's internal and external forces, inertia, or energy. Kinematics may be defined in terms of the vehicle's location, velocity, and acceleration [11]. Kinematic modeling is an essential method for determining the vehicle's lateral motion concerning its speed and acceleration, as well as its yaw motion. In addition to formulating the route tracking error between the planned and actual 
trajectory of the vehicle location, it is frequently used with dynamic models to develop controller algorithms.

Because the model assumes just one wheel per axle, it ignores the influence of slip from each wheel in the vehicle's direction, which is finally defined as:

$$
\left[\begin{array}{l}
x \\
y \\
\theta^{\prime}
\end{array}\right]=\left[\begin{array}{cc}
\cos \theta & 0 \\
\sin \theta & 0 \\
0 & 1
\end{array}\right]\left[\begin{array}{l}
v \\
\psi
\end{array}\right]
$$

The $\mathrm{x}$ represents longitudinal velocity, $y$ represents lateral velocity, $\theta$ is the vehicle's orientation, and represents the vehicle's yaw rate. Because longitudinal is considered constant and does not aid with route tracking guidance, only lateral or left/proper movement with yaw rate is required in the controller.

As reference point $x_{d}, y_{d}$, and $\theta_{d}$ may be acquired from the reference route established, getting the global coordinates of the location and heading orientation of the vehicle is also valuable for future prediction control or error computation. The vehicle's current location is also stored in $x_{c}, y_{c}$, and $\theta_{c}$, allowing for comparison and error computation when the reference point is subtracted. Matrix translation from global coordinate to vehicle local coordinate yields a longitudinal error, $x_{e}$, lateral error, $y_{e}$, and heading error [12-13] as in equation follow:

$$
\left[\begin{array}{l}
x_{e} \\
y_{e} \\
\theta_{e}
\end{array}\right]=\left[\begin{array}{ccc}
\cos \theta_{c} & \sin \theta_{c} & 0 \\
-\sin \theta_{c} & \cos \theta_{c} & 0 \\
0 & 0 & 1
\end{array}\right]\left[\begin{array}{l}
x_{d}-x_{c} \\
y_{d}-y_{c} \\
\theta_{d}-\theta_{c}
\end{array}\right]
$$

\section{CONTROL STRUCTURE}

The proposed path tracking control system will be modeled after the block design shown in Fig. 4. This block diagram's overall control system demonstrates that the first stage in developing the vehicle path tracking is to create a reference route or desired path using $\mathrm{x}$ and $y$ coordinates and a vehicle heading angle. Before feeding back to the lateral controller, the error computation between the intended path and the predicted vehicle location is assessed. Next, the nonlinear/linear lateral controller will estimate the appropriate steering wheel angle to achieve zero path error. The vehicle model, which includes both dynamic and kinematic characteristics, will then create vehicle motion with the proper sideslip angle and yaw rate. Consequently, the suggested control block diagram above is based on yaw rate monitoring. If the lateral error is significant, a larger steering angle is generated to rotate the position vehicle towards the intended route using a feedback closed-loop system using future prediction control.

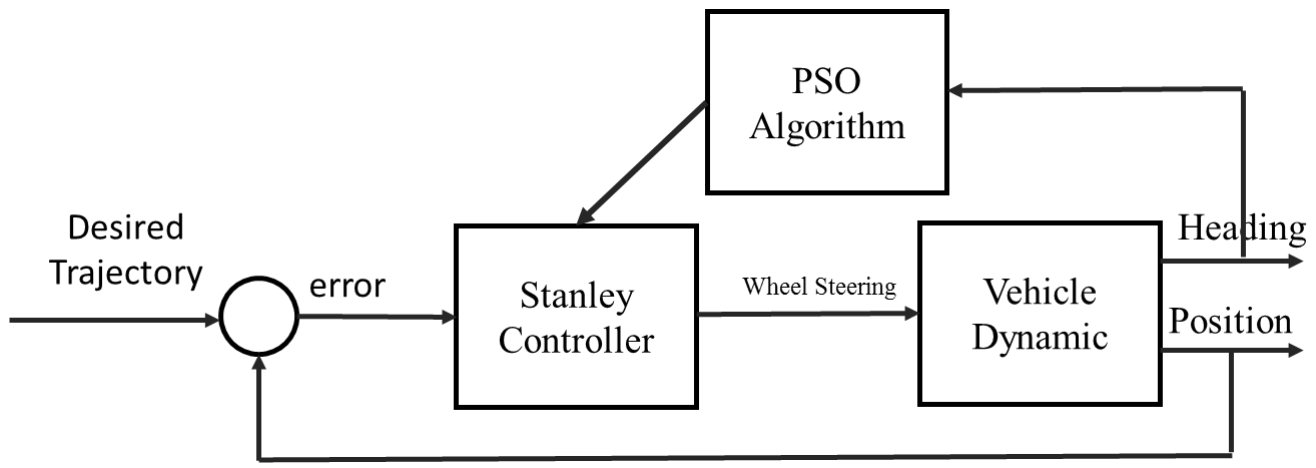

Fig. 4. Stanley controller with PSO algorithm for path tracking 
The particle swarm optimization technique is based on stochastic processes and is built around a population of organisms/particles. This method is based on a model of a live creature, such as flocks of birds. These creatures then interact based on social-psychological connections in the same manner as live species do, and they can adapt to diverse conditions.

In order to find the best solution, starting organisms/particles must be produced at random. The method follows an absolute path by utilizing solution particle location and particle velocity vectors as a guide. We may decide that a specific solution within a particular optimization period is determined by the velocity vector, which defines our best solution.

It is calculated as a fitness function, also known as the capacity to discover a better solution. This type of vector denotes the personal best values for each organism inside the system and is referred to as the unique best solution (pBest). In contrast, each particle swarm within every singular moment has its best global position called gBest.

Initial locations for each organism within the search space are created randomly, which is finally defined in equation (13). The algorithm then performs optimization cycles, with each iteration searching for the current personal best solution (pBest) and global best solution (gBest). Eq. 1 shows the core of the optimization algorithm, while Eq. 2 stands for updating the particle location after each optimization cycle.

$$
\begin{aligned}
& v_{i}=v_{i}+c_{1} \operatorname{rand}()\left(p_{i}-x_{i}\right)+c_{2} \operatorname{Rand}()\left(p_{g}-x_{i}\right) \\
& x_{i}=x_{i}+v_{i}
\end{aligned}
$$

\section{STANLEY CONTROLLER}

The Stanley Controller has demonstrated excellent data perception and path planning capabilities. In addition, it provides excellent vehicle steering in both forward and backward directions. Not only that, but tests have shown that, despite its simplicity, the Stanley Controller outperforms other controllers, outperforming the Pure Pursuit Controller with more excellent transient responsiveness and reduced overshoot. Stanley Controller previously won the DARPA Grand Challenge with an autonomous vehicle utilizing an intuitive steering control rule [12]. A nonlinear feedback function of the cross-track error (lateral error) is assessed from the front axle center to the nearest path point in the Stanley Controller technique, as shown in Fig. 5. The control law idea is preserved by steering the front wheels and maintaining them aligned with the provided path by calculating the steering angle and heading error [13-14]. As a result of the control law, the intended path tracking effect is achieved, with the conclusion that as the lateral error rises, the wheels are guided closer to the route.

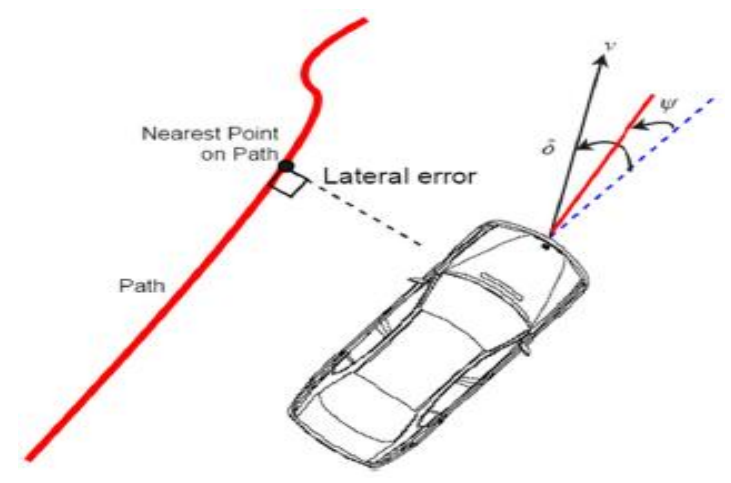

Fig. 5. Stanley controller design [4] 
The Stanley Controller, which is the most basic and widely used controller nowadays, is based on two properties: the heading error, which is the difference between the trajectory direction and the vehicle direction of motion, and the lateral error, which is determined by evaluating the orientation of the vehicle's trajectory path. The vehicle's instantaneous velocity is represented by $v$. The mathematical equation for Stanley Controller is [12]:

$$
\delta=\varphi+\tan ^{-1}\left(\frac{k e_{x}}{v}\right)
$$

\section{RESULTS}

Stanley controller performance analysis was carried out on the emergency trajectory type. Two-turn maneuvers are carried out by autonomous vehicles in a short time. The optimal controller gain is determined using the PSO algorithm. Determination of the optimal gain is calculated with the smallest lateral error RMSE fitness function.

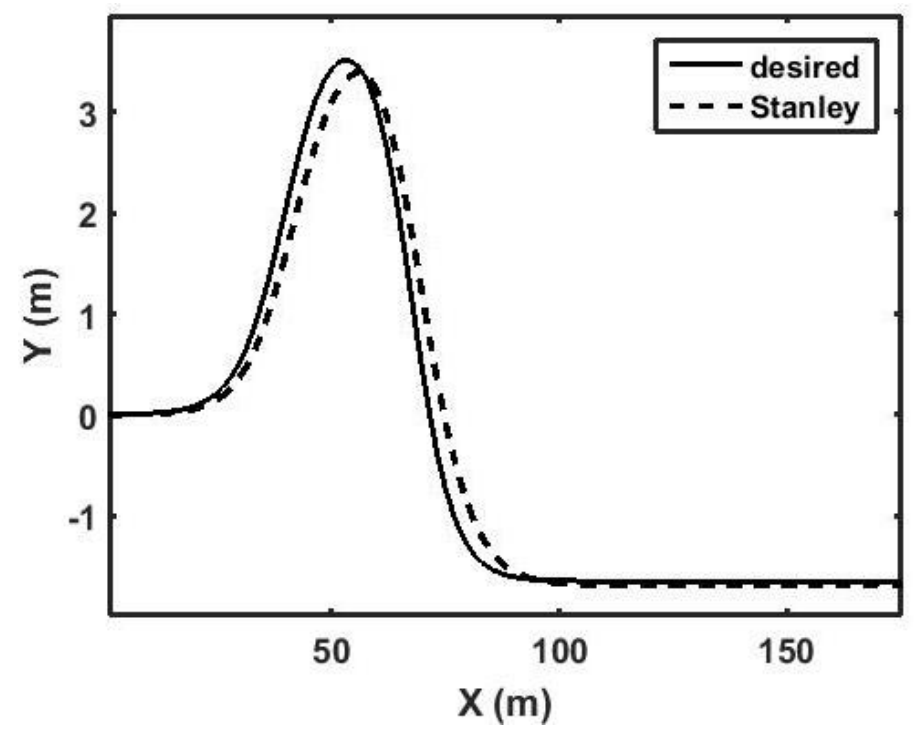

Fig. 6. Performance Stanley controller for the emergency trajectory of $20 \mathrm{~m} / \mathrm{s}$ vehicle speed

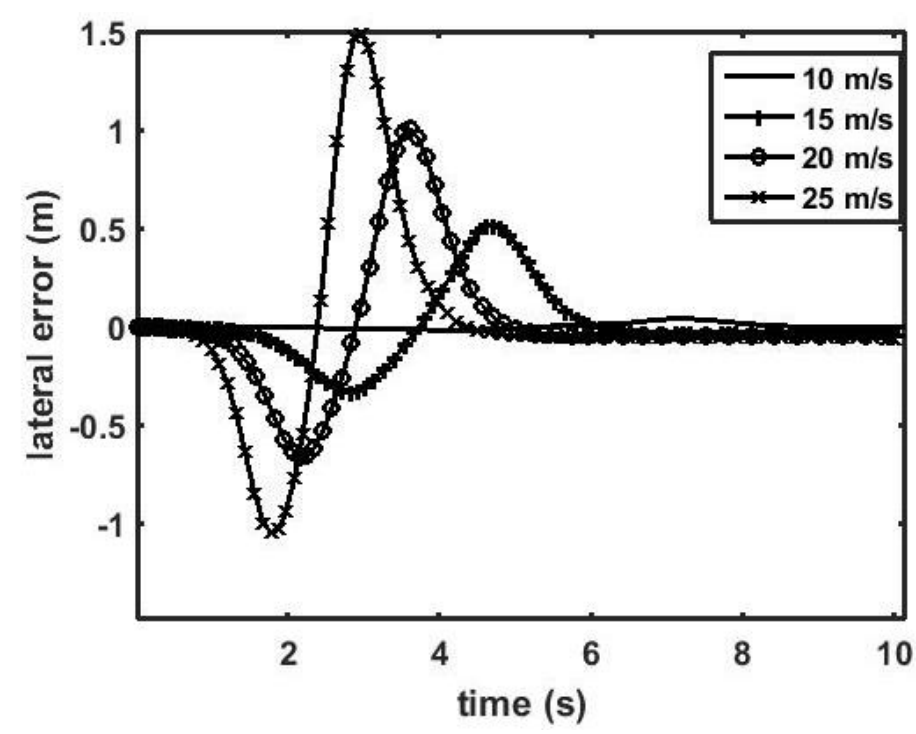

Fig. 7. Performance Stanley controller for lateral position tracking of various vehicle speed 


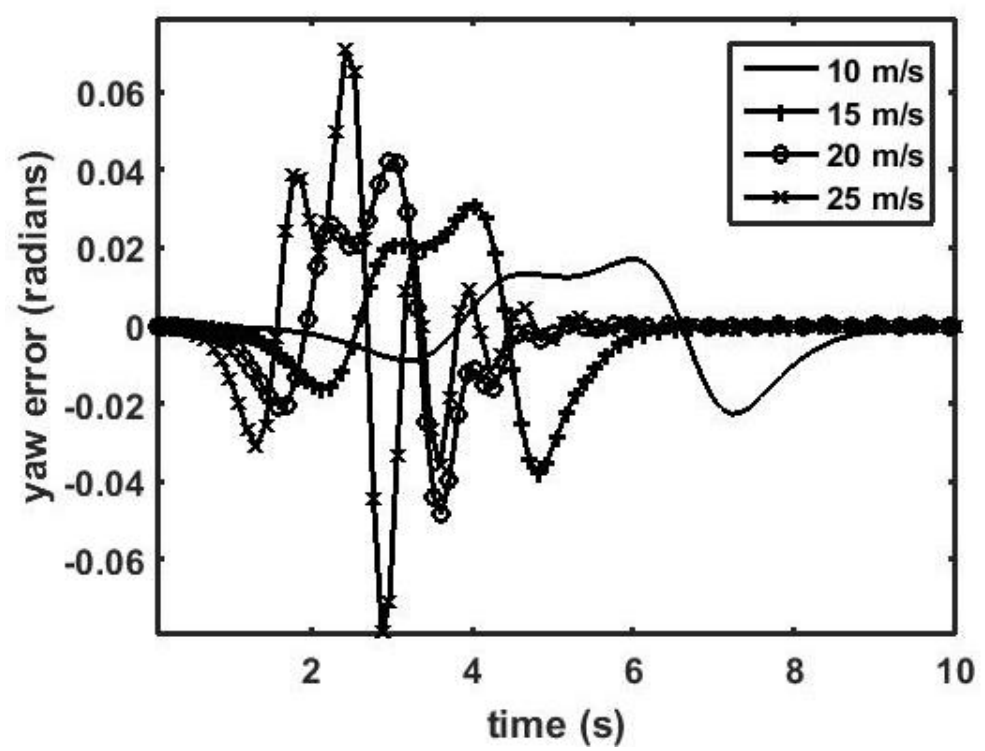

Fig. 8. Performance Stanley controller for yaw tracking of various vehicle speed

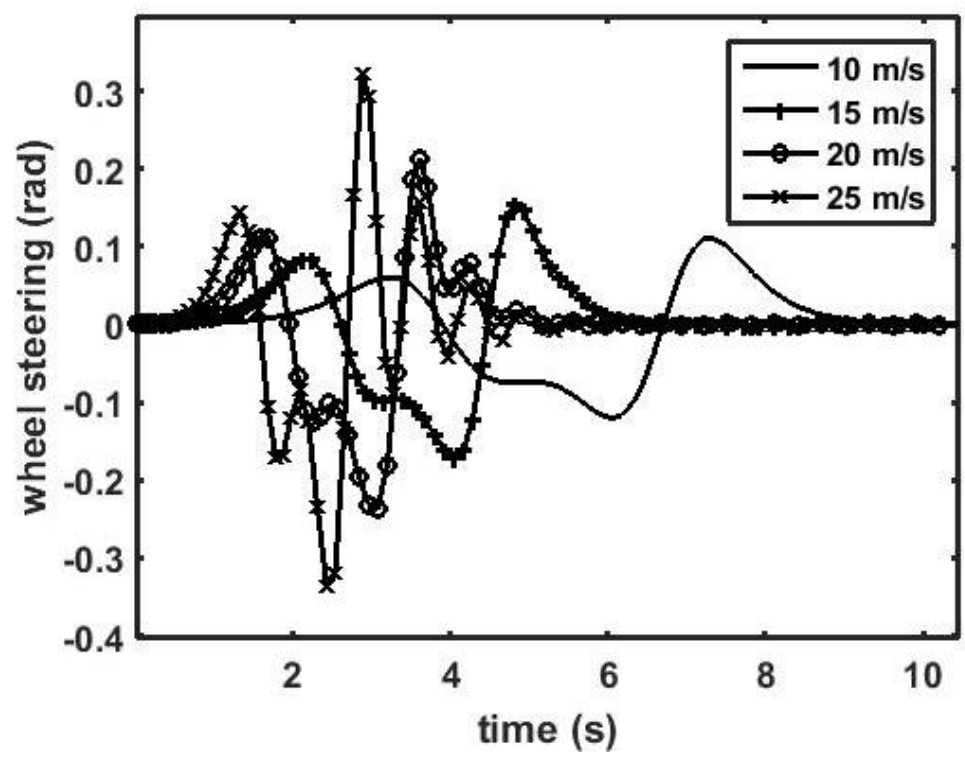

Fig. 9. Performance Stanley controller for steering of various vehicle speed

According to graphs, lateral position errors have increased with increasing the vehicle speed (Fig. 7). For example, there has been a rise in RMSE lateral position with the vehicle speed of $20 \mathrm{~m} / \mathrm{s}$. As a result, the error becomes $1.5 \mathrm{~m}$.

The highest yaw error was in vehicle speeds above $20 \mathrm{~m} / \mathrm{s}$ with road friction 1 (Fig. 8). As we can see on the graphs, yaw error increased significantly with vehicle speed above $20 \mathrm{~m} / \mathrm{s}$. Fig. 9 shows the steering wheel of the autonomous vehicle with various vehicle speeds. The aggressiveness of the steering indicated a vehicle speed of $25 \mathrm{~m} / \mathrm{s}$.

\section{CONCLUSIONS}

Overall, the Stanley approach excelled in most cases when the vehicle speed of the emergency trajectory was less than $20 \mathrm{~m} / \mathrm{s}$. The route tracking of the Stanley controller is precise with less steady-state error at varying speeds when the gain tuning parameter is set correctly. Aggressive maneuvering can be avoided by tuning the controller gain below the 
speed of $20 \mathrm{~m} / \mathrm{s}$. Further analysis can be performed by compression with other types of controllers. In addition, it can be done by analyzing various types of trajectories.

\section{REFERENCES}

[1] G. Han, W. Fu, W. Wang and Z. Wu, "The lateral tracking control for the intelligent vehicle based on adaptive PID neural network", Sensors, 17(6),2017, pp. 1 - 15.

[2] A. J. De Winter, "Design and Implementation of a Path Following System for an Autonomous Vehicle", Delft Center for Systems and Control, (1), 2017, pp. 7 - 72.

[3] X. Wang, M. Fu, H. Ma and Y. Yang, "Lateral control of autonomous vehicles based on fuzzy logic", Control Eng. Pract., 34, 2015, pp. 1 - 17.

[4] N. H. Amer, H. Zamzuri, K. Hudha and K. A. Kadir, "Modelling and Control Strategies in Path Tracking for Autonomous Ground Vehicles: A Review of State of the Art and Challenges", J. Intell. Robot. Syst. Theory. Appl., 86(2), 2017, pp. 225 - 254.

[5] M. A. Zakaria, H. Zamzuri and S. A. Mazlan, "Dynamic Curvature Steering Control for Autonomous Vehicle: Performance Analysis", IOP Conf. Ser. Mater. Sci. Eng., 114(1), 2016, pp. $1-8$.

[6] M. Hatta, H. Zamzuri, W. J. Yahya and S. A. Mazlan, "Optimal control strategy for low speed and high speed four - wheel - active steering vehicle", J. Mech. Eng. Sci., 8, 2015, pp. 1516 1528 .

[7] N. Hamzah, K. A. Danapalasingam, K. Peng, M. K. Aripin, M. F. Ismail and Y. Md Sam, "A Review of Active Yaw Control System for Vehicle Handling and Stability Enhancement", Int. J. Veh. Technol.,(1), 2014, pp. $1-15$.

[8] M. A. Zakaria, H. Zamzuri, S. A. Mazlan and S. M. H. F. Zainal, "Vehicle path tracking using future prediction steering control", Procedia Eng. 41, 2012, pp. 473 - 479.

[9] V. Milanes, J. E. Naranjo, C. Gonzalez, J. Alonso and T. Pedro, "Autonomous vehicle based in cooperative GPS and inertial systems", Robotica, 26(5), 2008, pp. $627-633$.

[10] J. Wang, J. Steiber and B. Surampudi, "Autonomous ground vehicle control system for high speed and safe operation", Proc. Am. Control Conf. (1), 2008, pp. 218 - 223

[11] A. De Luca, G. Oriolo and C. Samson, "Feedback control of a nonholomic car - like robot", In Robot Motion Planning and Control, (1), 1998, pp. 171 - 249.

[12] J. M. Snider, "Automatic Steering Methods for Autonomous Automobile Path Tracking", Robotics Institute Carnegie Mellon Uni., 8, 2009, pp. 8 -61.

[13] M. A. Zakaria, H. Zamzuri, R. Mamat and S. A. Mazlan, "A path tracking algorithm using future prediction control with spike detection for an autonomous vehicle robot", Int. J. Adv. Robot. Syst., 10, 2013, pp. $1-9$

[14] R. Solea and U. Nunes, "Trajectory Planning with Velocity Planner for Fully - automated Passenger Vehicles. Int", Transp. Syst. Conf. Toronto, 2, 2006, pp. $474-480$. 